\title{
MODELAMIENTO GEOMÉTRICO DEL CAMBIO DE COORDENADAS UTM CAUSADAS POR MUDANZA DE REFERENCIAL GEODÉSICO. CASO SIRGAS - CHILE
}

Geometric Modeling of Coordinate Transformation Between Geodetic Reference Systems in UTM Coordinate System. Case Sirgas - Chile

\author{
CARLOS MENA FRAU \\ LEONARDO MOLINA PINO \\ YONY ORMAZÁBAL ROJAS \\ YOHANA MORALES HERNÁNDEZ
}

Universidad de Talca, Centro de Geomática.

Avenida Lircay s/n. Talca, Chile.

[cmena, yormazabal, ymorales]@utalca.cl; lmolinapino@gmail.com

\begin{abstract}
RESUMEN
Los avances tecnológicos de las últimas décadas han permitido determinar cada vez con mayor precisión, las dimensiones, forma y campo gravitacional de la Tierra. Estas nuevas tecnologías y sus métodos de observación y análisis, admiten el uso de distintos Sistemas Geodésicos de Referencia (SGR) que hacen posible la descripción de posición de cualquier punto sobre la superficie terrestre con precisiones sub-métricas. Actualmente en Chile hay diversos SGR usados en la generación de productos cartográficos, tales como el Datum Provisorio Sudamericano de 1956 (PSAD56), el Datum Sudamericano de 1969 (SAD69) y el nuevo Sistema de Referencia Geocéntrico para las Américas (SIRGAS). Este último, a diferencia de los dos anteriores, es un sistema geocéntrico actual, compatible en precisión y exactitud con las modernas tecnologías de posicionamiento. Actualmente, el Instituto Geográfico Militar de Chile (IGM) promueve la adopción del nuevo sistema SIRGAS e indica el modelo y los parámetros oficiales de transformación para la cartografía nacional a escala 1:50.000. Con la adopción de SIRGAS, una cantidad significativa de documentos cartográficos, en pleno uso actualmente, deberán ser transformados o adecuados al nuevo referencial geodésico. Existen diversos métodos y modelos, propuestos en la geodesia, para la transformación de valores de coordenadas asociados a un SGR en
\end{abstract}


valores de coordenadas asociados a otro SGR. En el presente trabajo se realiza un análisis del impacto del cambio de referencial geodésico SIRGAS en la geometría de los rasgos representados en el sistema de proyección Universal Transversa de Mercator, para el caso de Chile continental sudamericano. Posteriormente, se definen modelos y parámetros alternativos al procedimiento clásico de transformación de coordenadas. Los resultados muestran que los modelos alternativos son eficaces en el caso de Chile.

Palabras-clave: Sistema Geodésico de Referencia; SIRGAS; Transformación de Coordenadas; Sistema de Coordenadas UTM.

\section{ABSTRACT}

Technological advances in recent decades have allowed determining with increasing precision, parameters related to size, shape and gravitational field of the Earth. These new technologies and methods of observation and analysis, accept the use of different Geodetic Reference Systems (GRS) that enable the description of position of any point on Earth's surface with sub-metric precision. Currently in Chile there are several SGR used in the generation of cartographic products, such as the Provisional South American Datum of 1956 (PSAD56), the South American Datum 1969 (SAD69) and the new Geocentric Reference System for the Americas (SIRGAS). The latter, unlike the previous two, is a recent geocentric system with precision and accuracy compatible with modern positioning technologies. Currently in Chile, the Military Geographical Institute (IGM) promotes the adoption of the new system and indicates the model and parameters of transformation to SIRGAS for national mapping at 1:50,000 scale. With the adoption of SIRGAS, a significant volume of cartographic documents in use nowadays must be transformed or adapted to the new geodetic reference. There are several methods and models proposed in geodesy to change geodetic coordinate values between different Geodetic Reference Systems. In this paper, a change impact analysis of geodetic reference SIRGAS in the geometry of the features represented in the Universal Transverse Mercator projection system, in the case of South American continental Chile, was realized. Subsequently, models and parameters are defined as alternatives to conventional process of coordinate transformation. The results show that alternative models are efficient in the Chile case.

Keywords: Geodetic Reference System; SIRGAS; Coordinate Transformation; UTM Coordinate System.

\section{INTRODUCCIÓN}

En Chile diversos sistemas geodésicos de referencia han sido adoptados como oficiales. En las últimas cinco décadas la red geodésica nacional ha sido establecida por dos sistemas clásicos locales simultáneamente, Datum Provisorio Sudamericano de 1956 (PSAD56) y Datum Sudamericano 1969 (SAD69). En el mapa base nacional escala 1:50.000 se ha utilizado PSAD56 entre los $17^{\circ} 30^{\prime}$ a los $43^{\circ} 30^{\prime}$ de 


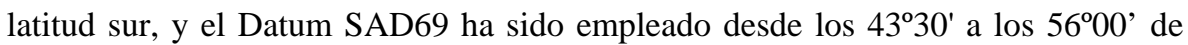
latitud sur, correspondiente a la zona austral del país. Actualmente, el Instituto Geográfico Militar de Chile (IGM) promueve la adopción del Sistema de Referencia Geocéntrico para las Américas (SIRGAS) en su realización año 2000, época 2002.0 (IGM, 2008).

Con la adopción de SIRGAS en el año 2002, se inicia un periodo de transición en el cual una gran cantidad de documentos cartográficos y bases de datos geográficos tendrán que ser transformados al nuevo referencial geodésico. La coexistencia de diversos sistemas y la implementación de SIRGAS, como nuevo referencial geodésico, demanda métodos y parámetros de transformación que atiendan las diversas necesidades de precisión en el uso particular de datos georreferenciados. En tanto, el Instituto Geográfico Militar (IGM), organismo responsable por el mapa base de la nación, indica un modelo y parámetros para la trasformación de coordenadas entre los sistemas vigentes, con una precisión nominal de $\pm 5 \mathrm{~m}$, para los productos cartográficos derivados del mapa base 1:50.000, publicado por dicho organismo. Sin embargo, aún no son divulgados los métodos y parámetros oficiales para la transformación entre todos los referenciales geodésicos a escalas mayores. En el presente documento se analiza el impacto del cambio de referencia geodésico en la geometría de la superficie proyectada en el sistema Universal Transversa de Mercator, sobre la base del modelo y parámetros divulgados por IGM (2008), para luego presentar dos modelos bidimensionales alternativos para la transformación de coordenadas.

\section{MODELO DE TRANSFORMACIÓN DE COORDENADAS MOLODENSKY}

No obstante que la geodesia aporta diversos modelos de transformación entre sistemas geodésicos de referencia (OLIVEIRA, 1998; CRIOLLO et al., 2005), el más utilizado en la actualidad es el modelo de Transformación de Similaridad (ANCIÃES y OLIVEIRA, 2003). Este modelo de transformación es conocido también como modelo de transformación Conforme, Isogonal, de Helmert (LUGNANI, 1987), o como modelo de transformación de Bursa/Wolf (RAPP, 1981).

Para el caso de Chile, IGM (2008) indica como modelo de transformación el modelo de Molodensky, y entrega además, un conjunto de parámetros para transformaciones entre SIRGAS y PSAD56, y entre SIRGAS y SAD69. Cada conjunto de parámetros corresponde a una zona geográfica de Chile (Tabla 1 y 2). El modelo de Molodensky transforma directamente coordenadas geodésicas, dadas en latitud, longitud y altura elipsoidal, por medio de incrementos en la componente horizontal y vertical, sin necesidad de una conversión intermedia de las coordenadas geodésicas al sistema geodésico cartesiano. El modelo de transformación es dado como sigue (NGA, 2011): 
donde,

$$
\begin{aligned}
& \phi_{2}=\phi_{1}+\Delta \phi \\
& \lambda_{2}=\lambda_{1}+\Delta \lambda \\
& h_{2}=h_{1}+\Delta h
\end{aligned}
$$

$\phi_{1}, \lambda_{1}, h_{1}$, son las coordenadas geodésicas en el sistema origen $S_{1}$

$\phi_{2}, \lambda_{2}, h_{2}$, son las coordenadas geodésicas en el sistema destino $S_{2}$

$\Delta \phi, \Delta \lambda, \Delta h$, son los incrementos dados por:

$$
\begin{gathered}
\Delta \phi=(M+h)^{-1}[-\Delta X \sin \phi \cos \lambda-\Delta Y \sin \phi \sin \lambda \\
+\Delta Z \cos \phi+\frac{\varepsilon^{2} \sin \phi \cos \phi}{\left(1-\varepsilon^{2} \sin ^{2} \phi\right)^{1 / 2}} \Delta a \\
\left.+\Delta f\left(M \frac{a}{b}+N \frac{a}{b}\right) \sin \phi \cos \phi\right] \\
\Delta \lambda=((N+h) \cos \phi)^{-1}[-\Delta X \sin \lambda+\Delta Y \cos \lambda] \\
\Delta h=\Delta X \cos \phi \cos \lambda+\Delta Y \cos \phi \sin \lambda \\
+\Delta Z \sin \phi-\Delta a\left(1-\varepsilon^{2} \sin ^{2} \phi\right)^{1 / 2} \\
+\Delta f \sin ^{2} \phi N(1-f)
\end{gathered}
$$

donde,

$-\Delta \phi, \Delta \lambda, \Delta h$, son las diferencias de coordenadas en los dos referenciales para un mismo punto,

$-\Delta X, \Delta Y, \Delta Z$ expresan la posición del origen del sistema $S_{2}$ en relación al sistema $S_{1}$,

$-\phi, \lambda, h$, son las coordenadas geodésicas correspondiente a latitud, longitud y altura elipsoidal respectivamente, del sistema de coordenadas geodésicas de origen, $M$ y $N$ son los radios de curvatura de la elipse meridiana y de la Gran Normal respectivamente, funciones de $a, b, \epsilon^{2}, f$, que son los parámetros de figura del elipsoide del sistema origen y corresponden respectivamente a los semi-ejes mayor y menor, primera excentricidad del elipsoide al cuadrado y achatamiento del elipsoide,

$-\Delta a$, es la diferencia de la magnitud del semieje mayor del elipsoide del sistema destino, respecto del semi-eje mayor del elipsoide del sistema origen,

Bol. Ciênc. Geod., sec. Artigos, Curitiba, v. 18, no 4, p.583-604, out-dez, 2012. 
$-\Delta f$, es la diferencia de la magnitud del achatamiento del elipsoide del sistema destino, respecto del achatamiento del elipsoide del sistema origen.

Se observa en las ecuaciones (1), (2) y (3) que el modelo considera paralelos, los sistemas geodésicos cartesianos involucrados, sin diferencias relativas de escala y no concéntricos. De esta forma, en la transformación son incluidos solamente las tres traslaciones $(\Delta X, \Delta Y, \Delta Z)$ del sistema geodésico cartesiano y las diferencias de parámetros de figura de los elipsoides, lo que puede ser considerado como una diferencia en escala.

Para la aplicación de la transformación de Molodensky, con el objeto de transformar coordenadas geodésicas que representan rasgos del terreno representados en un mapa o carta, en valores de coordenadas asociados a otro referencial geodésico, es necesario obtener, para cada punto que describe cada rasgo, los valores de coordenadas $(\phi, \lambda, h)$ en el sistema geodésico correspondiente. Los términos $(\phi, \lambda)$ son obtenidos directamente al aplicar, a las coordenadas de proyección de cada punto, las funciones inversas de la proyección cartográfica. El valor $h$, en general, no está contenido en la base cartográfica, y por tanto, debe ser deducido a partir de otros datos: altitud ortométrica $(H)$ y altitud geoidal $\left(N^{\prime}\right)$, en razón que $h \quad H+N^{\prime}$ (GEMAEL, 1999).

Tabla 1 - Parámetros de transformación de SIRGAS a PSAD56 (IGM, 2008).

\begin{tabular}{|c|c|c|}
\hline $\begin{array}{c}\text { Zona } 1 \\
\text { Intervalo } 17^{\circ} 30^{\prime} \mathrm{S} \text { a } 26^{\circ} 00^{\prime} \mathrm{S}\end{array}$ & $\begin{array}{c}\text { Zona } 2 \\
\text { Intervalo } 26^{\circ} 00^{\prime} \mathrm{S} \text { a } 36^{\circ} 00^{\prime} \mathrm{S}\end{array}$ & $\begin{array}{c}\text { Zona } 3 \\
\text { Intervalo } 36^{\circ} 00^{\prime} \mathrm{S} \text { a } 44^{\circ} 00 \mathrm{~S}^{\prime}\end{array}$ \\
\hline$\Delta \mathrm{X}=302 \mathrm{~m}$ & $\Delta \mathrm{X}=328 \mathrm{~m}$ & $\Delta \mathrm{X}=352 \mathrm{~m}$ \\
\hline$\Delta \mathrm{Y}=-272 \mathrm{~m}$ & $\Delta \mathrm{Y}=-340 \mathrm{~m}$ & $\Delta \mathrm{Y}=-403 \mathrm{~m}$ \\
\hline$\Delta \mathrm{Z}=360 \mathrm{~m}$ & $\Delta \mathrm{Z}=329 \mathrm{~m}$ & $\Delta \mathrm{Z}=287 \mathrm{~m}$ \\
\hline
\end{tabular}

Para transformar de PSAD56 a SIRGAS se debe invertir los signos de las translaciones

Tabla 2 - Parámetros de transformación de SIRGAS a SAD69 (IGM, 2008).

\begin{tabular}{c|c|c|c}
\hline $\begin{array}{c}\text { Zona } 1 \\
\text { Intervalo } \\
17^{\circ} 30^{\prime} \mathrm{S} \text { a 26 } 00^{\circ} \mathrm{S}\end{array}$ & Zona 2 & Zona 3 & Zona 4 \\
\cline { 2 - 4 } & $\begin{array}{c}\text { Intervalo } \\
26^{\circ} 00^{\prime} \mathrm{S} \text { a 36 } 00^{\circ} \mathrm{S}\end{array}$ & $\begin{array}{c}\text { Intervalo } \\
36^{\circ} 00^{\prime} \mathrm{S} \text { a 44 } 04^{\circ} \mathrm{S}\end{array}$ & $\begin{array}{c}\text { Intervalo } \\
44^{\circ} 00^{\prime} \mathrm{S} \text { a 56 } 00^{\circ}\end{array}$ \\
\hline$\Delta \mathrm{X}=59 \mathrm{~m}$ & $\Delta \mathrm{X}=64 \mathrm{~m}$ & $\Delta \mathrm{X}=72 \mathrm{~m}$ & $\Delta \mathrm{X}=79 \mathrm{~m}$ \\
\hline$\Delta \mathrm{Y}=11 \mathrm{~m}$ & $\Delta \mathrm{Y}=0 \mathrm{~m}$ & $\Delta \mathrm{Y}=-10 \mathrm{~m}$ & $\Delta \mathrm{Y}=-13 \mathrm{~m}$ \\
\hline$\Delta \mathrm{Z}=52 \mathrm{~m}$ & $\Delta \mathrm{Z}=32 \mathrm{~m}$ & $\Delta \mathrm{Z}=32 \mathrm{~m}$ & $\Delta \mathrm{Z}=14 \mathrm{~m}$ \\
\hline
\end{tabular}

Para transformar de SAD69 a SIRGAS se debe invertir los signos de las translaciones

La altitud ortométrica para cada rasgo representado en la base cartográfica puede ser obtenida a partir de las curvas de nivel en un proceso de interpolación. El 
valor de la altitud geoidal $\left(N^{\prime}\right)$ puede ser obtenido de mapas de altitudes geoidales de la región mapeada. Aunque este procedimiento no presenta ninguna complicación desde el punto de vista matemático, en la práctica si presenta complicaciones. Por un lado, la cantidad de cálculos del proceso de transformación aumenta significativamente por cuenta del procedimiento de interpolación, que busca la estimación del valor $H$ para cada punto que define espacialmente cada rasgo mapeado, y por otro lado, en algunos casos no hay disponibilidad de mapas de altitudes geoidales para determinar el valor $N^{\prime}$. Ante la imposibilidad de determinar la componente altimétrica $(h)$ se usa, para efecto de la transformación, considerar la altitud elipsoidal como igual a cero $(h=0)$, o caracterizar el conjunto de datos como bidimensional. Una evaluación de estos procedimientos es abordado por Anciães Y Oliveira (2003); Pino y Firkowski (2009).

Tabla 3 - Parámetros de elipsoides en Sistemas Geodésicos de Referencia

\begin{tabular}{c|c|c|c}
\hline $\begin{array}{c}\text { Sistema } \\
\text { Geodésico de } \\
\text { Referencia }\end{array}$ & elipsoide & semieje mayor (a) & achatamiento (f) \\
\hline PSAD56 & $\begin{array}{c}\text { Internacional } \\
1924\end{array}$ & $6.378 .388 \mathrm{~m}$ & $1 / 297$ \\
\hline SAD69 & $\begin{array}{c}\text { Internacional } \\
1967\end{array}$ & $6.378 .160 \mathrm{~m}$ & $\begin{array}{c}1 / 298,247167427 \\
\text { aproximado al valor } \\
1 / 298,25\end{array}$ \\
\hline SIRGAS & GRS80 & $6.378 .137 \mathrm{~m}$ & $1 / 298,257222101$ \\
\hline
\end{tabular}

\section{SISTEMA DE COORDENADAS UNIVERSAL TRANSVERSA DE MERCATOR}

El sistema de coordenadas Universal Transversa de Mercator (UTM) es un sistema derivado por especificación de la proyección cartográfica Transversa de Mercator (TM). El sistema UTM surgió en la década del 40 y fue desarrollado por la U.S. Army, como respuesta a la necesidad de una proyección global para propósitos militares. Los requisitos para la proyección fueron minimizar la distorsión del valor de los azimut y mantener la distorsión de escala dentro de límites pre-establecidos (SMITH, 1996).

La proyección cartográfica Transversa de Mercator (TM) es una representación conforme del elipsoide, y predomina sobre otros tipos de proyecciones cartográficas utilizadas, con excepción de las proyecciones conformes utilizadas para la representación de las zonas polares, para las cuales se utiliza la proyección estereográfica polar (BLACHUT et al., 1979).

Las fórmulas para el cálculo de coordenadas y de otras cantidades de la proyección TM, pueden ser consultadas en Snyder (1987), Pearson (1990) y Blachut et al. (1979). En este trabajo serán utilizadas las fórmulas dadas en Blachut et al. 
(1979). Por razones prácticas y en razón de mantener uniformidad en el contexto del presente artículo, el eje $x$ será orientado en sentido Este y el eje $y$ en el sentido Norte.

Figura 1 - Distorsión de escala para cuadrante Sur-Este de un huso UTM.

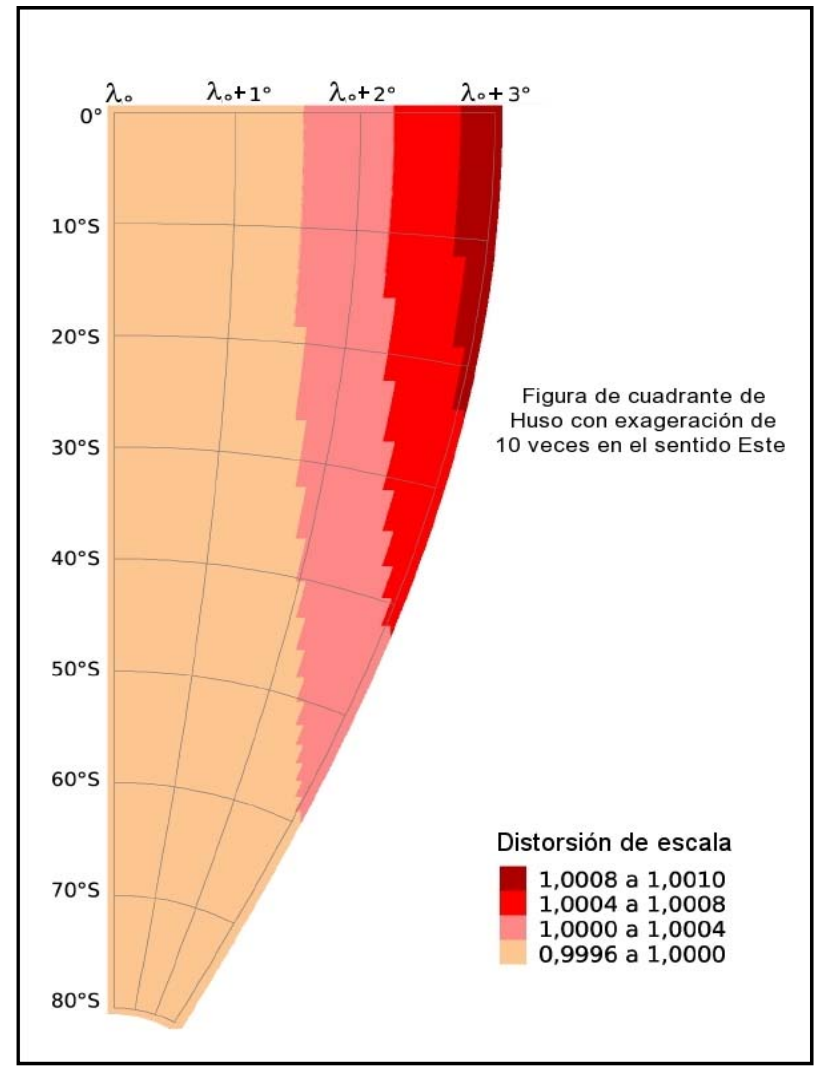

\subsection{Comportamiento de la distorsión de escala $m$}

El comportamiento del valor de la distorsión de escala en un huso UTM puede ser observado en la Figura 1. El valor que representa la distorsión de escala aumenta directamente con la distancia al meridiano central, esto es, un valor de distorsión de escala es de 0,9996 para puntos localizados en el meridiano central, y valores mayores para puntos apartados del meridiano central. Así, en la faja central del huso UTM, próximo al meridiano central, el valor de distorsión de escala es menor que 1 , y fuera de ella mayor que 1. Por tanto, el huso UTM presenta dos líneas sin distorsión de escala, que corresponde a las líneas límites de dicha faja central. De 
esta forma, y considerando que los cambios de referencial geodésico tienen un efecto en los valores de coordenadas, las variaciones en longitud tendrán un efecto mayor en la distorsión de escala, que las variaciones de latitud, y aún mayores en los bordes del huso. Además, variaciones observadas en los valores de longitud geodésica, tienen un efecto mayor en las zonas cercanas al ecuador que variaciones en igual magnitud en zonas australes. Obsérvase en la Figura 1 que el comportamiento tiene una tendencia de variación paralela al eje central del huso y por tanto, cuando las variaciones en longitud son observadas en su correspondiente valor en la componente $E$ UTM, la variación de la distorsión de escala tiende a ser la constante para una misma magnitud de variación $E$ UTM, para cualquier zona equidistante al eje del huso UTM. Para el caso de variaciones en la componente latitudinal o en la componente $N$ UTM, el valor de la distorsión de escala tiende a mantenerse, especialmente en zonas cercanas al eje central del huso.

Figura 2 - Convergencia meridiana en el cuadrante Sur-Este de un huso UTM.

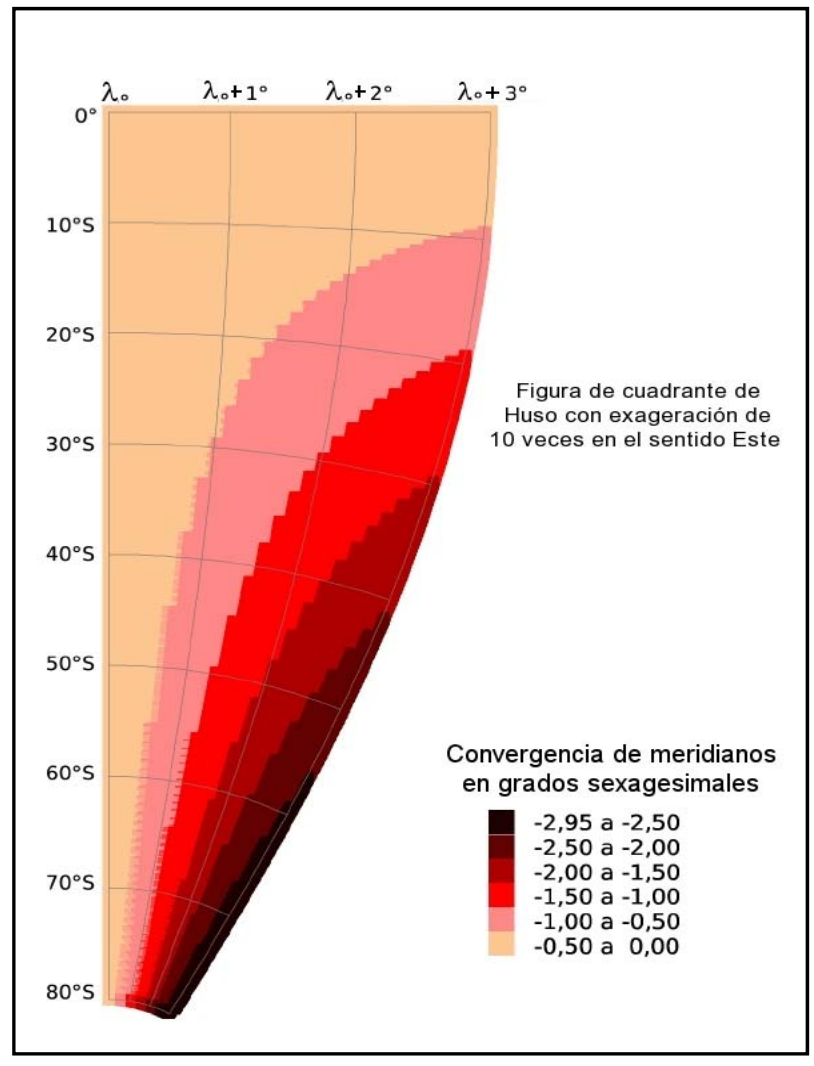

Bol. Ciênc. Geod., sec. Artigos, Curitiba, v. 18, no 4, p.583-604, out-dez, 2012. 


\subsection{Comportamiento de la Convergencia Meridiana}

El comportamiento del valor de la convergencia meridiana puede ser observado en la Figura 2. El valor de la convergencia meridiana, en valor absoluto, aumenta con la latitud y con la diferencia en longitud al meridiano central, es decir, puntos localizados en las proximidades de la latitud $0^{\circ} \mathrm{y}$ en las proximidades del meridiano central presentan, en valor absoluto, magnitudes de convergencia meridiana menores al observado en puntos localizados en latitudes altas y en longitudes extremas del huso. Los menores valores de convergencia meridiana, en valor absoluto, se ubican en zonas cercanas al Ecuador y al meridiano central del huso. Considerando que los cambios de referencial geodésico tienen un efecto en los valores de coordenadas, las variaciones en longitud tendrán un efecto mayor, en el valor de convergencia meridiana, cuando éstas se presentan en zonas australes, y menores cuando se presentan en zonas cercanas al Ecuador. Por otro lado, las variaciones en la componente latitudinal tendrán un efecto mayor en la convergencia meridiana si estas variaciones se presentan en las zonas cercanas al Ecuador y cercanas al borde del huso.

\section{MÉTODOLOGÍA}

\subsection{Obtención de valores de coordenadas para la determinación del modelo y} parámetros de transformación

Para determinar el impacto del cambio de referencial geodésico en la geometría de los rasgos representados en el sistema de proyección UTM, se aplica el modelo y parámetros de transformación dados por IGM (2008). Luego se analizan las magnitudes de las variaciones de las coordenadas Norte y Este UTM, distorsión de escala y convergencia meridiana. Este análisis se fundamenta en el hecho que un cambio de referencial geodésico produce valores de coordenadas de proyección distintos a los valores originales, lo que puede ilustrarse como un desplazamiento aparente del punto observado, y por lo tanto, el punto asume nuevos valores para la distorsión de escala y de convergencia meridiana. En una segunda etapa son modeladas las variaciones mencionadas anteriormente con el auxilio de modelos de transformación existentes y de características bidimensionales. Finalmente, y con el propósito de validar la aplicabilidad de los modelos bidimensionales, son obtenidos los parámetros propios en un proceso de ajuste por el método de los mínimos cuadrados y luego son analizadas sus precisiones y valores de residuos.

Los datos sintéticos son un conjunto de 18 puntos distribuidos a lo largo y ancho del territorio de Chile continental sudamericano (Tabla 4), tal que, a lo menos cuatro puntos estén contenidos en cada zona de parámetros definidas en IGM (2008). En razón de considerar situaciones máximas extremas en relación a las diferencias en valores de coordenadas transformadas versus coordenadas originales, se aplicó los parámetros de transformación de PSAD56 a SIRGAS para las zonas 1, 2 y 3. Para la zona austral (zona 4) se utilizó los parámetros SAD69 a SIRGAS, en razón de que para la zona austral no existe cobertura en PSAD56. 
Al conjunto de datos sintéticos les es aplicado el modelo de transformación y parámetros dados por IGM (2008). El resultado es un conjunto de coordenadas transformadas al nuevo referencial geodésico, las que a su vez son transformadas a coordenadas en el sistema UTM (Tabla 5). El desplazamiento aparente de cada punto evaluado sobre el sistema UTM es de magnitud aproximada a 400m en dirección Sur-Oeste (Figura 3), para las zonas 1, 2 y 3. Para la zona 4 la magnitud del desplazamiento aparente de los puntos alcanza valores próximos a los $70 \mathrm{~m}$. Estos valores, notoriamente menores a los obtenidos en las zonas 1, 2 y 3, tienen explicación por la mayor proximidad del centro del sistema geodésico de referencia SAD69 con respecto al SIRGAS y un mejor alineamiento de ejes, que el correspondiente PSAD56, lo que además queda en evidencia en los parámetros de transformación, cuyos valores son menores, en valor absoluto, a 80m.

Tabla 4 - Valores de coordenadas geodésicas (Longitud $\lambda$ y Latitud $\phi$ ) del sistema origen. Valores angulares en grados sexagesimales.

\begin{tabular}{|c|c|c|c|c|c|}
\hline ID & Huso & Zona & $\lambda$ & $\phi$ & Datum \\
\hline 1 & 18 & $\mathrm{Z3}$ & $-73,0$ & $-38,0$ & \multirow{3}{*}{ PSAD56 } \\
\hline 2 & 18 & $\mathrm{Z3}$ & $-74,0$ & $-43,0$ & \\
\hline 3 & 18 & $\mathrm{Z3}$ & $-72,5$ & $-43,0$ & \\
\hline 4 & 18 & $\mathrm{Z4}$ & $-75,5$ & $-48,5$ & \multirow{2}{*}{ SAD69 } \\
\hline 5 & 18 & $\mathrm{Z4}$ & $-73,0$ & $-48,5$ & \\
\hline 6 & 19 & $\mathrm{Z} 1$ & $-70,0$ & $-20,0$ & \multirow{11}{*}{ PSAD56 } \\
\hline 7 & 19 & $\mathrm{Z1}$ & $-68,6$ & $-20,0$ & \\
\hline 8 & 19 & $\mathrm{Z} 1$ & $-70,0$ & $-25,0$ & \\
\hline 9 & 19 & $\mathrm{Z} 1$ & $-68,6$ & $-25,0$ & \\
\hline 10 & 19 & $\mathrm{Z1}$ & $-70,0$ & $-23,5$ & \\
\hline 11 & 19 & $\mathrm{Z1}$ & $-67,5$ & $-23,5$ & \\
\hline 12 & 19 & $\mathrm{Z} 2$ & $-71,0$ & $-30,0$ & \\
\hline 13 & 19 & $\mathrm{Z} 2$ & $-70,0$ & $-30,0$ & \\
\hline 14 & 19 & $\mathrm{Z} 2$ & $-71,5$ & $-35,0$ & \\
\hline 15 & 19 & $\mathrm{Z} 2$ & $-70,5$ & $-35,0$ & \\
\hline 16 & 19 & $\mathrm{Z3}$ & $-71,5$ & $-38,0$ & \\
\hline 17 & 19 & $\mathrm{Z} 4$ & $-71,0$ & $-55,0$ & \multirow{2}{*}{ SAD69 } \\
\hline 18 & 19 & $\mathrm{Z} 4$ & $-68,0$ & $-55,0$ & \\
\hline
\end{tabular}


Tabla 5 - Valores de coordenadas UTM en el sistema origen (1), coordenadas UTM en el sistema destino (2) y valor de desplazamiento aparente de los puntos $(d S)$. Valores lineales en metros.

\begin{tabular}{c|c|c|c|c|c}
\hline ID & Este (1) & Norte (1) & Este (2) & Norte (2) & $\boldsymbol{d} \boldsymbol{S}$ \\
\hline 1 & $675.611,226$ & $5.792 .227,803$ & $675.375,213$ & $5.791 .864,238$ & 433,453 \\
\hline 2 & $581.512,393$ & $5.238 .614,309$ & $581.276,111$ & $5.238 .253,814$ & 431,029 \\
\hline 3 & $703.784,860$ & $5.236 .066,364$ & $703.547,557$ & $5.235 .705,699$ & 431,731 \\
\hline 4 & $463.063,997$ & $4.627 .984,950$ & $462.991,148$ & $4.627 .970,326$ & 74,302 \\
\hline 5 & $647.740,617$ & $4.626 .174,059$ & $647.667,441$ & $4.626 .159,429$ & 74,624 \\
\hline 6 & $395.386,386$ & $7.788 .178,666$ & $395.202,072$ & $7.787 .853,692$ & 373,604 \\
\hline 7 & $541.844,069$ & $7.788 .440,971$ & $541.659,647$ & $7.788 .116,036$ & 373,623 \\
\hline 8 & $399.082,747$ & $7.234 .642,805$ & $398.898,923$ & $7.234 .319,599$ & 371,824 \\
\hline 9 & $540.365,787$ & $7.234 .955,469$ & $540.181,170$ & $7.234 .632,298$ & 372,187 \\
\hline 10 & $397.892,013$ & $7.400 .739,051$ & $397.708,045$ & $7.400 .415,029$ & 372,605 \\
\hline 11 & $653.168,656$ & $7.400 .294,780$ & $652.983,630$ & $7.399 .970,702$ & 373,177 \\
\hline 12 & $307.076,619$ & $6.679 .482,358$ & $306.892,811$ & $6.679 .107,212$ & 417,756 \\
\hline 13 & $403.545,710$ & $6.680 .745,476$ & $403.361,658$ & $6.680 .370,473$ & 417,735 \\
\hline 14 & $271.835,854$ & $6.124 .039,615$ & $271.653,475$ & $6.123 .667,430$ & 414,468 \\
\hline 15 & $363.111,099$ & $6.125 .867,903$ & $362.927,951$ & $6.125 .495,881$ & 414,661 \\
\hline 16 & $280.479,845$ & $5.791 .165,659$ & $280.295,632$ & $5.790 .788,444$ & 419,792 \\
\hline 17 & $372.071,340$ & $3.903 .357,922$ & $372.002,492$ & $3.903 .338,695$ & 71,482 \\
\hline 18 & $563.967,657$ & $3.904 .729,926$ & $563.898,482$ & $3.904 .710,702$ & 71,797 \\
\hline
\end{tabular}

En cuanto a las variaciones del valor de distorsión de escala (Tabla 6) éstas son menores o iguales a 1ppm, dado principalmente por la dirección del desplazamiento aparente del punto, el cual es levemente ortogonal a la razón de cambio máximo de la distorsión de escala para las zonas 1, 2 y 3. De igual forma, las diferencias del valor de la convergencia meridiana, no alcanza valores mayores a los 7 ” $\left(0,0019^{\circ}\right)$. Las variaciones de los valores $m$ y $C$ para la zona 4 son pequeñas debido principalmente a que los desplazamientos aparentes de los puntos son pequeños (>75m) en relación a la razón de cambio de ambos parámetros, pese a que los desplazamientos aparentes son relativamente en la misma dirección de la razón de cambio máxima. 
Figura 3 - Desplazamiento aparente de puntos por cambio de referencial geodésico.

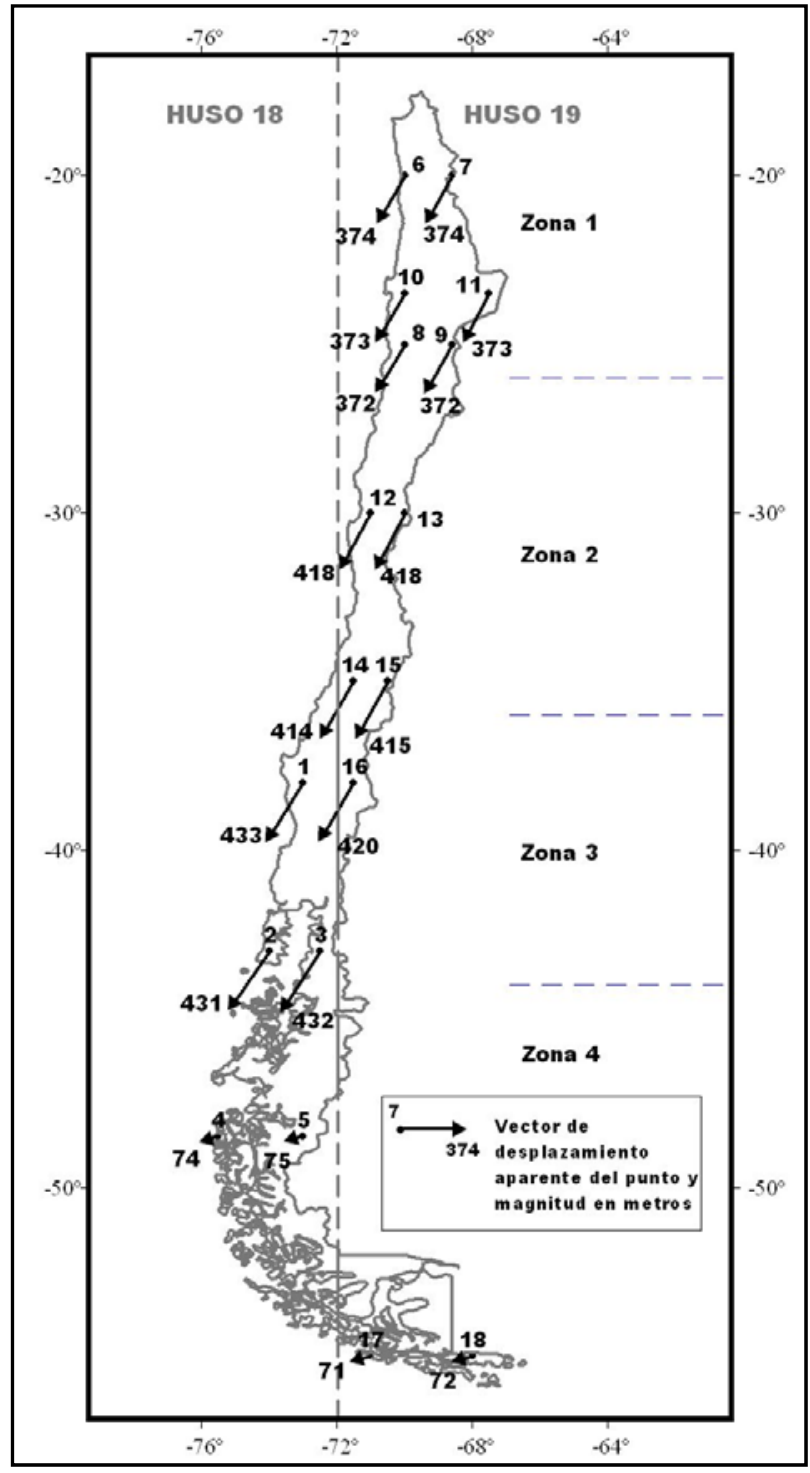

Bol. Ciênc. Geod., sec. Artigos, Curitiba, v. 18, nº 4, p.583-604, out-dez, 2012. 
Tabla 6 - Valores de distorsión de escala $m$ y convergencia meridiana $C$ en el sistema origen (1) y en el sistema destino (2) para los puntos utilizados en el análisis. Valores angulares expresados en el sistema sexagesimal.

\begin{tabular}{|c|c|c|c|c|c|c|}
\hline \multirow[b]{2}{*}{ ID } & \multirow[b]{2}{*}{$m(1)$} & \multirow[b]{2}{*}{$m(2)$} & \multirow[b]{2}{*}{$m(2)-m(1)$} & \multicolumn{2}{|c|}{ Grados } & \multirow{2}{*}{$\begin{array}{l}\text { Segundos } \\
\mathrm{C}(2)-C(1)\end{array}$} \\
\hline & & & & $C(1)$ & $C(2)$ & \\
\hline 1 & 0,999980 & 0,999979 & $-9,900 \mathrm{E}-07$ & $-1,231637$ & $-1,230211$ & 5,13522 \\
\hline 2 & 0,999682 & 0,999681 & $-4,700 \mathrm{E}-07$ & $-0,682036$ & $-0,680186$ & 6,65975 \\
\hline 3 & 1,000111 & 1,000110 & $-1,150 \mathrm{E}-06$ & $-1,705581$ & $-1,703915$ & 5,99854 \\
\hline 4 & 0,999617 & 0,999617 & $0,000 \mathrm{E}+00$ & 0,375226 & 0,375226 & 0,00000 \\
\hline 5 & 0,999868 & 0,999868 & $0,000 \mathrm{E}+00$ & $-1,497461$ & $-1,497461$ & 0,00000 \\
\hline 6 & 0,999735 & 0,999736 & $4,800 \mathrm{E}-07$ & 0,342051 & 0,342727 & 2,43302 \\
\hline 7 & 0,999622 & 0,999621 & $-1,900 \mathrm{E}-07$ & $-0,136810$ & $-0,136236$ & 2,06572 \\
\hline 8 & 0,999726 & 0,999726 & 4,700E-07 & 0,422654 & 0,423504 & 3,06068 \\
\hline 9 & 0,999620 & 0,999620 & $-1,800 \mathrm{E}-07$ & $-0,169050$ & $-0,168308$ & 2,66818 \\
\hline 10 & 0,999729 & 0,999729 & 4,700E-07 & 0,398784 & 0,399580 & 2,86780 \\
\hline 11 & 0,999890 & 0,999889 & $-6,800 \mathrm{E}-07$ & $-0,598241$ & $-0,597635$ & 2,17904 \\
\hline 12 & 1,000059 & 1,000060 & $9,000 \mathrm{E}-07$ & 1,000309 & 1,001459 & 4,13694 \\
\hline 13 & 0,999715 & 0,999715 & $4,500 \mathrm{E}-07$ & 0,500039 & 0,501091 & 3,78900 \\
\hline 14 & 1,000242 & 1,000243 & $1,070 \mathrm{E}-06$ & 1,434560 & 1,435977 & 5,09875 \\
\hline 15 & 0,999831 & 0,999832 & $6,300 \mathrm{E}-07$ & 0,860498 & 0,861812 & 4,72853 \\
\hline 16 & 1,000194 & 1,000195 & $1,040 \mathrm{E}-06$ & 1,539768 & 1,541350 & 5,69592 \\
\hline 17 & 0,999801 & 0,999801 & $2,200 \mathrm{E}-07$ & 1,638524 & 1,639434 & 3,27341 \\
\hline 18 & 0,999650 & 0,999650 & $-1,100 \mathrm{E}-07$ & $-0,819180$ & $-0,818308$ & 3,13783 \\
\hline
\end{tabular}

\subsection{Modelamiento del impacto geométrico del cambio de referencial geodésico}

El cambio de sistema geodésico de referencia tiene un impacto directo en la geometría de los rasgos representados en el sistema de proyección UTM, debido a la variación del valor de las coordenadas geodésicas que son variables de la función que determina las coordenadas en la proyección de dichos rasgos. Un cambio de valor de coordenadas implica, además, un cambio en las cantidades que caracterizan la deformación lineal y orientación, dados por el módulo de distorsión de escala y por la convergencia meridiana, respectivamente.

El desplazamiento aparente de los puntos y las diferencias observadas en convergencia meridiana y distorsión de escala en la proyección pueden ser modelados de la siguiente forma. Sea un punto $P$ sobre un huso cualquiera del 
sistema UTM (Figura 4) y $P^{\prime}$ la representación del mismo punto en el sistema UTM para el caso en que sus coordenadas geodésicas son transformadas a otro referencial geodésico. Sea $C$ y $C^{\prime}$ el valor de la convergencia meridiana en el punto $P$ y $P^{\prime}$, respectivamente. Además, sea $d S$ un segmento infinitesimal en el punto $P$ y $d S^{\prime}$ la proyección del segmento $d S$ posterior al cambio de referencial geodésico. El cambio en los valores de coordenadas del punto $P$ puede ser conceptualizado como el desplazamiento aparente de punto en el sistema de proyección, y por lo tanto, modelado como la traslación del origen del sistema de referencia cartesiano de la proyección, en el sentido de las abscisas $(\Delta E)$ y en las ordenadas $(\Delta N)$. La variación de la convergencia meridiana puede ser conceptualizada como una rotación en el punto $P$, cuya magnitud está dada por la diferencia entre la convergencia meridiana en el punto $P$ y la convergencia meridiana en el punto $P^{\prime}\left(C^{\prime}-C\right)$. La diferencia entre la distorsión de escala $(m)$ observada en el punto $P$ y la observada en el punto $P^{\prime}$, puede ser modelada por medio de un factor de escala cuya magnitud es dada por la razón entre los segmentos infinitesimales $\left(d S^{\prime} / d S\right)$.

Figura 4 - Desplazamiento aparente de un punto $P$ en un sector de huso UTM, convergencia meridiana $C$ y segmento infinitesimal $d S$.

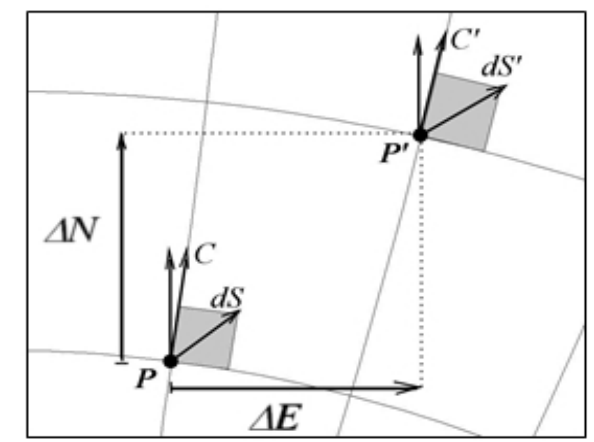

Dada una cobertura cartográfica de alguna zona en estudio, si la cobertura es de un tamaño suficientemente pequeño, y está en una posición tal que la razón de cambio de la convergencia meridiana y la razón de cambio de la distorsión de escala pueden ser modelados linealmente, entonces, es posible que dichos efectos sean modelados por medio de un modelo de transformación conforme o de similaridad. Este modelo de transformación es dado por las siguientes ecuaciones, que corresponden al modelo de Trasformación de Similaridad 2D:

$$
\begin{aligned}
& E_{2}-E_{c}=\Delta E+\left(E_{1}-E_{c}\right) k \cos \theta+\left(N_{1}-N_{c}\right) k \sin \theta \\
& N_{2}-N_{c}=\Delta N-\left(E_{1}-E_{c}\right) k \sin \theta+\left(N_{1}-N_{c}\right) k \cos \theta
\end{aligned}
$$


donde, $E_{2}, N_{2}$ son las coordenadas del punto observadas en el sistema UTM asociado al referencial geodésico destino; $E_{l}, N_{l}$ son las del punto observados en el sistema UTM asociado al referencial geodésico origen; $\Delta E$ y $\Delta N$ son los valores de traslación del sistema en el eje $x$ e $y$ respectivamente; $k$ es el factor de escala aplicado al sistema; y $\theta$ es el ángulo de rotación del sistema. $E_{c}, N_{c}$ son las coordenadas UTM de un centroide para la región donde ocurre la transformación. Esto es necesario dado que la rotación $\theta$ ocurre en el punto $P$ y no en el origen de las coordenadas UTM, pues en caso de aplicar la rotación en el origen de las coordenadas UTM, y debido a la exagerada magnitud de sus valores en comparación al pequeño valor angular de rotación, los efectos de dicha rotación generan, en la práctica, solamente traslaciones en el sentido de las coordenadas $E$ y no las rotaciones esperadas.

El modelo puede ser linearizado considerando $k \cos \theta$ como un valor $a$, y $k \sin \theta$ como un valor $b$, quedando la expresión de la siguiente forma:

$$
\begin{aligned}
& E_{2}-E_{c}=a\left(E_{1}-E_{c}\right)+b\left(N_{1}-N_{c}\right)+\Delta E \\
& N_{2}-N_{c}=-b\left(E_{1}-E_{c}\right)+a\left(N_{1}-N_{c}\right)+\Delta N
\end{aligned}
$$

Por otro lado, si se consideran las ecuaciones propias del sistema de proyección UTM, las variaciones en los valores de coordenadas por cambios de referencial geodésico, pueden ser modeladas por variaciones en los parámetros definitorios del sistema UTM para cada huso. Así, la traslación $\Delta E$ puede ser modelada por la variación del valor Falso Este $(F E)$, la traslación $\Delta N$ por la variación del Falso Norte $(F N)$, la rotación, dada por la variación de la convergencia meridiana, puede ser modelada por variación del valor del meridiano central del Huso $\left(\lambda_{0}\right)$, finalmente, la variación de distorsión de escala puede ser modelada por la variación del valor del factor de escala $m_{0}$, impuesto al meridiano central $\left(\lambda_{0}\right)$. De esta manera, el modelo queda expresado como sigue:

$$
\begin{aligned}
& E_{2}=F E+m_{0}\left[a_{1}\left(\lambda-\lambda_{0}\right)+a_{3}\left(\lambda-\lambda_{0}\right)^{3}+a_{5}\left(\lambda-\lambda_{0}\right)^{5}\right] \\
& N_{2}=F N+m_{0}\left[B+a_{2}\left(\lambda-\lambda_{0}\right)^{2}+a_{4}\left(\lambda-\lambda_{0}\right)^{4}+a_{6}\left(\lambda-\lambda_{0}\right)^{6}\right]
\end{aligned}
$$

donde, $E_{2}, N_{2}$ son las coordenadas cartesianas bidimensionales del punto en el sistema UTM asociado al referencial geodésico destino; $F E, F N, m_{0} \mathrm{y} \lambda_{0}$ son, respectivamente, los parámetros del huso UTM modificado Falso Este, Falso Norte, Factor de escala y valor de la longitud del meridiano central; $\lambda$ es el valor de la longitud del punto asociado al referencial geodésico origen. Los demás coeficientes son los propios de la proyección Transversa de Mercator.

Todos los algoritmos necesarios para realizar los experimentos fueron implementados en el lenguaje Octave (GNU Octave, version 3.2.2), lenguaje de alto nivel orientado al tratamiento de computo numérico. 


\section{EXPERIMENTOS}

\subsection{Obtención de los parámetros propios para cada modelo}

Para la obtención de los parámetros en cada modelo fue aplicado un ajuste por medio del método paramétrico. El desarrollo completo de este método puede ser consultado en MIKHAIL (1976), GEMAEL (1994), y en DALMOLIN (2004).

Dado que los modelos propuestos constan de cuatro parámetros cada uno, tres puntos son suficientes para resolver el ajuste con dos grados de libertad, puesto que cada punto aporta dos ecuaciones al sistema. No obstante, para obtener mayor grado de libertad se consideraron cuatro puntos por cada zona de parámetros IGM (Figura 6). Dado que el modelo UTM modificado no es lineal, los parámetros deben ser obtenidos mediante iteraciones, con inicio en un conjunto de parámetros arbitrarios. Para el caso en estudio se utilizó los valores $500.000 \mathrm{~m}, 10.000 .000 \mathrm{~m}$ y 1,0000 para $F E, F N, m_{0}$, respectivamente y, dependiendo del huso, $-75^{\circ}$ ó $-69^{\circ}$ para $\lambda_{0}$.

Los resultados del proceso de ajuste aportaron los parámetros para cada zona y huso UTM, tanto para el modelo de Similaridad como para la UTM modificada (Tabla 7 y Tabla 8). La Tabla 9 muestra las diferencias entre los parámetros obtenidos y los valores nominales del huso UTM. Puede observarse que los valores de los parámetros obtenidos para ambos modelos son similares en magnitud y dirección para las zonas 1, 2 y 3. La zona 4, tanto para el huso 18 como para el huso 19, presentaron valores, para la componente Norte, significativamente diferentes en magnitud y dirección.

Dado que en el modelo de similaridad $a=k \cos \theta$ y $b=k \sin \theta$, se deduce entonces que las rotaciones son significativamente pequeñas, menores a 0,512 segundos, y por tanto el valor del parámetro $a$ es, en la práctica, igual al valor de parámetro de escala $k$. Esto significa que el modelo puede ser simplificado, para el caso en estudio, como un modelo de similaridad con rotaciones nulas y afecto solamente por translaciones en la componente $E$ y en la componente $N$, y un factor de escala aplicado a ambas componentes.

Las precisiones de los parámetros en el modelo de similaridad presentan valores pequeños, menores a $0,43 \mathrm{ppm}$ para los parámetros $a$ y $b$ y sub-métricos para las translaciones $\Delta E$ y $\Delta N$. Dentro de éstos, los de mayor magnitud se presentan en la zona 4 con magnitud de $0,515 \mathrm{~m}$ y $0,620 \mathrm{~m}$ para el huso 19 y 18 respectivamente. En tanto, para el modelo UTM modificado, la situación de las translaciones $\Delta E$ y $\Delta N$ se presenta en forma inversa con valores mayores para las zonas 1,2 y 3 , y magnitudes menores para la zona 4 (menores a $0,218 \mathrm{~m}$ ). Las precisiones para el factor de escala del meridiano central son menores a $0,31 \mathrm{ppm}, \mathrm{y}$ la precisión para los valores de longitud son menores a 0,17 segundos sexagesimales. 
Tabla 7 - Parámetros obtenidos para la transformación de similaridad 2D en cada zona y huso.

\begin{tabular}{c|r|r|r|r|r|r}
\hline & \multicolumn{7}{c|}{ Huso 19 } & \multicolumn{2}{c}{ Huso 18 } \\
\cline { 2 - 7 } & Zona 1 & Zona 2 & \multicolumn{1}{c}{ Zona 3 } & \multicolumn{1}{c}{ Zona 4 } & Zona 3 & Zona 4 \\
\hline \multicolumn{7}{c}{ Valores de parámetros } \\
\hline a & 0,999997 & 0,999995 & 1,000001 & 0,999961 & 0,999995 & 0,999963 \\
\hline $\mathbf{b}$ & $-2,70 \mathrm{E}-07$ & $-1,73 \mathrm{E}-06$ & $-2,48 \mathrm{E}-06$ & $9,40 \mathrm{E}-07$ & $1,90 \mathrm{E}-06$ & $3,50 \mathrm{E}-07$ \\
\hline$\Delta \mathbf{E}$ (metros) & $-184,293$ & $-183,354$ & $-184,191$ & $-67,307$ & $-236,375$ & $-75,493$ \\
\hline$\Delta \mathbf{N}$ (metros) & $-324,072$ & $-373,586$ & $-377,085$ & 86,546 & $-362,085$ & 77,895 \\
\hline \multicolumn{7}{c}{ Precisión de los parámetros } \\
\hline a & $4,30 \mathrm{E}-07$ & $3,50 \mathrm{E}-07$ & $4,40 \mathrm{E}-07$ & $3,11 \mathrm{E}-06$ & $3,80 \mathrm{E}-07$ & $3,40 \mathrm{E}-06$ \\
\hline b & $4,30 \mathrm{E}-07$ & $3,50 \mathrm{E}-07$ & $4,40 \mathrm{E}-07$ & $3,11 \mathrm{E}-06$ & $3,80 \mathrm{E}-07$ & $3,40 \mathrm{E}-06$ \\
\hline$\Delta \mathbf{E}$ (metros) & 0,124 & 0,098 & 0,069 & 0,515 & 0,107 & 0,620 \\
\hline$\Delta \mathbf{N}$ (metros) & 0,124 & 0,098 & 0,069 & 0,515 & 0,107 & 0,620 \\
\hline
\end{tabular}

Residuos de los valores de coordenadas ajustadas (metros)

\begin{tabular}{r|r|r|r|r|r|r}
\hline $\mathbf{E}_{\mathbf{1}}$ & $-0,172$ & $-0,134$ & 0,080 & $-0,777$ & $-0,178$ & $-0,811$ \\
\hline $\mathbf{E}_{\mathbf{2}}$ & 0,179 & 0,143 & $-0,016$ & $-0,431$ & 0,103 & $-0,738$ \\
\hline $\mathbf{E}_{\mathbf{3}}$ & 0,172 & 0,133 & 0,020 & 0,528 & 0,184 & 0,747 \\
\hline $\mathbf{E}_{\mathbf{4}}$ & $-0,179$ & $-0,142$ & $-0,084$ & 0,680 & $-0,109$ & 0,802 \\
\hline $\mathbf{N}_{\mathbf{1}}$ & $-0,003$ & 0,006 & $-0,024$ & 0,462 & 0,037 & $-0,371$ \\
\hline $\mathbf{N}_{\mathbf{2}}$ & 0,002 & $-0,007$ & $-0,079$ & $-0,390$ & $-0,028$ & 0,440 \\
\hline $\mathbf{N}_{\mathbf{3}}$ & $-0,001$ & $-0,009$ & 0,119 & $-0,358$ & $-0,011$ & 0,375 \\
\hline $\mathbf{N}_{\mathbf{4}}$ & 0,002 & 0,010 & $-0,016$ & 0,286 & 0,002 & $-0,444$ \\
\hline
\end{tabular}

Precisión de los valores de coordenadas ajustadas (metros)

\begin{tabular}{l|l|l|l|r|r|r}
\hline $\mathbf{E}_{\mathbf{1}}$ & 0,175 & 0,139 & 0,090 & 0,719 & 0,153 & 0,892 \\
\hline $\mathbf{E}_{\mathbf{2}}$ & 0,176 & 0,137 & 0,118 & 0,768 & 0,149 & 0,862 \\
\hline $\mathbf{E}_{\mathbf{3}}$ & 0,175 & 0,137 & 0,069 & 0,658 & 0,150 & 0,873 \\
\hline $\mathbf{E}_{\mathbf{4}}$ & 0,176 & 0,139 & 0,092 & 0,753 & 0,151 & 0,879 \\
\hline $\mathbf{N}_{\mathbf{1}}$ & 0,175 & 0,139 & 0,090 & 0,719 & 0,153 & 0,892 \\
\hline $\mathbf{N}_{\mathbf{2}}$ & 0,176 & 0,137 & 0,118 & 0,768 & 0,149 & 0,862 \\
\hline $\mathbf{N}_{\mathbf{3}}$ & 0,175 & 0,137 & 0,069 & 0,658 & 0,150 & 0,873 \\
\hline $\mathbf{N}_{\mathbf{4}}$ & 0,176 & 0,139 & 0,092 & 0,753 & 0,151 & 0,879 \\
\hline
\end{tabular}


Tabla 8 - Parámetros obtenidos para la UTM modificada en cada zona y huso.

\begin{tabular}{|c|c|c|c|c|c|c|}
\hline & \multicolumn{4}{|c|}{ Huso 19} & \multicolumn{2}{|c|}{ Huso 18} \\
\hline & Zona 1 & Zona 2 & Zona 3 & Zona 4 & Zona 3 & Zona 4 \\
\hline \multicolumn{7}{|c|}{ Valores de parámetros } \\
\hline $\begin{array}{c}\text { FE } \\
\text { metros }\end{array}$ & 499819,813 & 499833,047 & 499836,205 & 499931,162 & 499750,302 & 499926,825 \\
\hline $\begin{array}{c}\text { FN } \\
\text { metros }\end{array}$ & 9999668,008 & 9999606,786 & 9999625,255 & 9999971,215 & 9999613,993 & 9999975,162 \\
\hline $\begin{array}{c}\lambda_{0} \\
\text { grados }\end{array}$ & $-68,9999591$ & $-68,9998157$ & $-68,9997687$ & $-68,9999965$ & $-75,0001677$ & $-75,0000036$ \\
\hline $\mathbf{m}_{\mathbf{0}}$ & 0,999597 & 0,999594 & 0,9996 & 0,999598 & 0,999595 & 0,999598 \\
\hline \multicolumn{7}{|c|}{ Precisión de los parámetros } \\
\hline $\begin{array}{c}\text { FE } \\
\text { metros }\end{array}$ & 4,724 & 2,448 & 2,549 & 0,172 & 1,981 & 0,168 \\
\hline $\begin{array}{c}\text { FN } \\
\text { metros }\end{array}$ & 0,769 & 0,883 & 1,292 & 0,218 & 1,193 & 0,173 \\
\hline $\begin{array}{c}\lambda_{0} \\
\text { seg. }\end{array}$ & 0,16544851 & 0,09388818 & 0,10403092 & 0,00937231 & 0,08419796 & 0,00840855 \\
\hline $\mathbf{m}_{\mathbf{0}}$ & 0,00000031 & 0,00000024 & 0,00000031 & 0,00000004 & 0,00000027 & 0,00000003 \\
\hline
\end{tabular}

\begin{tabular}{c|r|r|r|r|r|r}
\hline \multicolumn{7}{c}{ Residuos de los valores de coordenadas ajustadas (metros) } \\
\hline $\mathbf{E}_{\mathbf{1}}$ & $-0,172$ & $-0,133$ & 0,081 & $-0,013$ & $-0,177$ & 0,012 \\
\hline $\mathbf{E}_{\mathbf{2}}$ & 0,179 & 0,143 & $-0,012$ & 0,011 & 0,102 & $-0,010$ \\
\hline $\mathbf{E}_{\mathbf{3}}$ & 0,172 & 0,133 & 0,014 & $-0,010$ & 0,184 & 0,010 \\
\hline $\mathbf{E}_{\mathbf{4}}$ & $-0,179$ & $-0,142$ & $-0,083$ & 0,012 & $-0,109$ & $-0,012$ \\
\hline $\mathbf{N}_{\mathbf{1}}$ & $-0,004$ & 0,001 & $-0,020$ & $-0,001$ & 0,043 & 0,001 \\
\hline $\mathbf{N}_{\mathbf{2}}$ & 0,004 & $-0,001$ & $-0,080$ & $-0,003$ & $-0,031$ & $-0,001$ \\
\hline $\mathbf{N}_{\mathbf{3}}$ & 0,001 & $-0,003$ & 0,120 & 0,001 & $-0,017$ & $-0,001$ \\
\hline $\mathbf{N}_{\mathbf{4}}$ & $-0,001$ & 0,004 & $-0,020$ & 0,003 & 0,006 & 0,001 \\
\hline
\end{tabular}

Precisión de los valores de coordenadas ajustadas (metros)

\begin{tabular}{l|l|l|l|l|l|l}
\hline $\mathbf{E}_{\mathbf{1}}$ & 0,124 & 0,098 & 0,063 & 0,008 & 0,108 & 0,008 \\
\hline $\mathbf{E}_{\mathbf{2}}$ & 0,124 & 0,097 & 0,084 & 0,009 & 0,105 & 0,008 \\
\hline $\mathbf{E}_{\mathbf{3}}$ & 0,124 & 0,097 & 0,049 & 0,008 & 0,106 & 0,008 \\
\hline $\mathbf{E}_{\mathbf{4}}$ & 0,124 & 0,098 & 0,065 & 0,009 & 0,107 & 0,008 \\
\hline $\mathbf{N}_{\mathbf{1}}$ & 0,124 & 0,098 & 0,063 & 0,008 & 0,108 & 0,008 \\
\hline $\mathbf{N}_{\mathbf{2}}$ & 0,124 & 0,097 & 0,084 & 0,009 & 0,105 & 0,008 \\
\hline $\mathbf{N}_{\mathbf{3}}$ & 0,124 & 0,097 & 0,049 & 0,008 & 0,106 & 0,008 \\
\hline $\mathbf{N}_{\mathbf{4}}$ & 0,124 & 0,098 & 0,065 & 0,009 & 0,107 & 0,008 \\
\hline
\end{tabular}

Bol. Ciênc. Geod., sec. Artigos, Curitiba, v. 18, nº 4, p.583-604, out-dez, 2012. 
Tabla 9 - Diferencias entre los parámetros obtenidos y los valores nominales del huso UTM.

\begin{tabular}{c|c|c|c|c|c|c}
\hline \multirow{2}{*}{} & \multicolumn{5}{|c|}{ Huso 19 } & \multicolumn{2}{c}{ Huso 18 } \\
\cline { 2 - 7 } & Zona 1 & Zona 2 & Zona 3 & Zona 4 & Zona 3 & Zona 4 \\
\hline $\begin{array}{c}\Delta \mathbf{F E} \\
\text { metros }\end{array}$ & $-180,187$ & $-166,953$ & $-163,795$ & $-68,838$ & $-249,698$ & $-73,175$ \\
\hline $\begin{array}{c}\Delta \mathbf{F N} \\
\text { metros }\end{array}$ & $-331,992$ & $-393,214$ & $-374,745$ & $-28,785$ & $-386,007$ & $-24,838$ \\
\hline $\begin{array}{c}\Delta \boldsymbol{\lambda}_{\mathbf{0}} \\
\text { grados }\end{array}$ & 0,0000409 & 0,0001843 & 0,0002313 & 0,0000035 & $-0,0001677$ & $-0,0000036$ \\
\hline$\Delta \mathbf{m}_{\mathbf{0}}$ & $-0,000003$ & $-0,000006$ & 0,000000 & $-0,000002$ & $-0,000005$ & $-0,000002$ \\
\hline
\end{tabular}

Los residuos obtenidos, así como las precisiones de los valores ajustados, resultaron ser todos sub-métricos. Los puntos ubicados en la zona 4 en el huso 18 y 19 obtuvieron los mayores valores de residuos y desviaciones estándar de valores ajustados en el modelo de similaridad, mientras que en el modelo UTM modificado estos mismos puntos presentaron magnitudes menores. Además, se observa una estrecha semejanza entre los dos modelos para las zonas 1, 2, y 3, con magnitudes en general, del orden de los decímetros para la componente $E$ y del orden de los centímetros para la componente $N$. Nuevamente se observan diferencias en la zona 4 al comparar ambos sistemas. En el modelo de similaridad las magnitudes de los residuos se presentan mayores en la zona 4, mientras que en el modelo UTM modificado, en esta misma zona, las magnitudes de los residuos resultan ser las menores.

En cuanto a las precisiones de los valores de coordenadas ajustadas, todas ellas resultan ser sub-métricas. Sus magnitudes siguen la tendencia observada en los residuos, a saber, para el modelo de similaridad los mayores valores se presentan en la zona 4, mientras que en el modelo UTM modificado, esta misma zona presenta las magnitudes menores. Se destaca que en el modelo UTM modificado, las magnitudes de las precisiones obtenidas para los valores de coordenadas ajustadas en la zona 4 son inferiores a $0,010 \mathrm{~m}$.

No obstante las pequeñas magnitudes observadas en los valores de coordenadas ajustadas, se debe advertir que éstas provienen de un ajuste a partir de datos sintéticos, los cuales son obtenidos por una transformación cuya aplicación entrega valores de coordenadas con precisión de \pm 5 metros, y por tanto las coordenadas ajustadas deben ser consideradas a lo menos con exactitudes iguales a las iniciales del experimento, es decir, \pm 5 metros.

En general, los modelos evaluados presentan magnitudes de precisiones y de residuos de los valores de coordenadas ajustadas en las zonas 1, 2 y 3 . En cuanto a las precisiones de los parámetros el modelo de similaridad presenta mejor 
desempeño. Para la zona 4, el modelo UTM modificado presenta magnitudes menores de valores de precisión y de residuos, como también los correspondientes valores de precisión de los parámetros. Dado que la zona 4 está en el intervalo $44^{\circ} 00^{\prime} \mathrm{S}$ a $56^{\circ} 00^{\prime} \mathrm{S}$, esta zona es la que presenta mayor razón de cambio para la convergencia meridiana como para la distorsión de escala, en la dirección del desplazamiento aparente de los puntos evaluados. Así, el modelo UTM modificado resulta ser el que mejor se ajusta a dicha zona, modelando con eficacia tales característica geométricas.

Desde el punto de vista del cómputo, la aplicación del modelo UTM modificado para la transformación de datos requiere menor esfuerzo, ya que es aplicado directamente a los valores de coordenadas geodésicas originales y como resultado se obtiene los valores de coordenadas en el sistema UTM, asociados al sistema geodésico de destino. El modelo de similaridad requiere de la definición de un centroide, y por lo tanto, los valores de coordenadas deben ser referidos a un sistema cartesiano intermedio antes de realizar el ajuste. En consecuencia el modelo de similaridad debe ser descrito por los cuatro parámetros que lo definen más las coordenadas del centroide utilizado.

\section{CONCLUSIONES}

En el presente trabajo fueron realizados experimentos para evaluar el impacto del cambio de referencial geodésico en la geometría de los rasgos representados en el sistema de proyección UTM, específicamente para el caso de Chile continental sudamericano, y en el contexto del nuevo referencial geodésico SIRGAS. Además, fueron evaluados dos modelos bidimensionales para la transformación de coordenadas al nuevo referencial geodésico. La metodología consistió en generar un cambio de referencial geodésico a puntos sintéticos distribuidos a través del territorio en estudio, según modelo y parámetros oficiales dados por IGM (2008). A partir de estos resultados se analizó las variaciones en las coordenadas UTM, y sus efectos en la distorsión de escala y en el valor de la convergencia meridiana. Luego, y a partir de este análisis se homologaron estos efectos a sistemas bidimensionales de transformación de coordenadas, el modelo de similaridad y el modelo que considera la modificación de los parámetros definitorios del huso UTM. Finalmente, fueron evaluados los dos modelos bidimensionales mediante un proceso de ajuste por el método paramétrico, utilizando valores de coordenadas referidos al sistema original PSAD56 y SAD69 y valores de coordenadas en el sistema SIRGAS. Los resultados evidenciaron que dado el modelo de transformación IGM (2008), las variaciones de coordenadas entre los sistemas geodésicos tienen efectos que pueden ser modelados como traslación, rotación y escalamiento de los rasgos representados en el sistema UTM, y por lo tanto, modelar estas variaciones a través de un sistema bidimensional conforme. La aplicación del ajuste por el método paramétrico entre los valores de coordenadas asociados a los sistemas geodésicos involucrados en el estudio y proyectados en el sistema UTM, permitieron validar los modelos bidimensionales propuestos como satisfactorios, a escalas donde los valores 
submétricos de residuos y de precisión de valores ajustados no son perceptibles. No obstante, el modelo de similaridad presentó mejor desempeño para las zonas 1, 2 y 3 , zonas caracterizadas, a diferencia de la zona 4, por presentar una razón de cambio de la convergencia meridiana y de la distorsión de escala, menos pronunciada. Para la zona 4, donde la razón de cambio de la convergencia meridiana y de la distorsión de escala es mayor para la dirección del desplazamiento aparente de los puntos evaluados, el modelo UTM modificado resultó ser el que mejor se ajusta a dicha zona, modelando con eficacia tales características geométricas. En cuanto al esfuerzo computacional, el modelo UTM modificado, en comparación al modelo de similaridad, presenta ventajas debido a que puede ser aplicado directamente a las coordenadas geodésicas de origen, con un esfuerzo computacional menor.

Finalmente, cabe observar que el disponer de conjuntos de parámetros distintos para cada zona, pudiera generar conflictos en la articulación de productos cartográficos por problemas de vecindad. Por cuestiones de tiempo, este problema no fue abordado por los autores, no obstante, queda propuesto como continuidad de investigación.

\section{BIBLIOGRAFÍA}

ANCIÃES, C. L. C.; OLIVEIRA, L. C. de. Transformação entre redes geodésicas: considerações sobre os espaços $3 D$ e 2D. Série em Ciências Geodésicas, v.3, pp. 85-104, 2003.

BLACHUT, T. J.; CHRZANOWSKI, A.; SAASTAMOINEN, J. H. Cartografía y Levantamientos Urbanos, New York: Springer-Verlag, 1979. 519 p.

CRIOLLO, A. R. T.; DALAZOANA, R.; DE FREITAS, S. R. C. O uso de redes neurais artificiais para melhorar a transformação de coordenadas entre redes geodésicas de referência clássicas. In: IV Colóquio Brasileiro de Ciências Geodésicas, 2005, Curitiba. Anais do IV Colóquio Brasileiro de Ciências Geodésicas. Curitiba: CPGCG, 2005. v. 1. p. 1-6.

DALMOLIN, Q. Ajustamento por Mínimos Quadrados, Segunda Edição. Curitiba: Imprensa Universitária - UFPR, 2004. 176 p.

GEMAEL, C. Introdução à Geodésia Física. Curitiba: Editora UFPR, 1999. 304 p.

GEMAEL, C. Introdução ao ajustamento de observações: aplicações geodésicas. Curitiba: Editora UFPR, 1994. 319 p.

IGM - Instituto Geográfico Militar. Nuevo marco de referencia geodésico. RGN SIRGAS-CHILE. 2008, 62 p.

LUGNANI, J. B. Introdução à Fototriangulação. Curitiba: Impresso na Imprensa Universitária da UFPR, 1987, 134 p.

MIKHAIL, E. M. Observations and least squares. New York: IEP, 1976, 497 p.

NGA - National Geospatial-Intelligence Agency. Coordinate System Analysis, Datum Transformations. Disponible en: <http://earth-info.nga.mil/GandG /coordsys/datums/index.html> acceso en 20/09/2011. 
OLIVEIRA, L. C. de. Realizações do Sistema Geodésico Brasileiro Associadas ao SAD 69 - Uma Proposta Metodológica de Transformação. São Paulo, 1998, 197 p. Tese (Doutorado em Engenharia). Escola Politécnica da Universidade de São Paulo.

PEARSON, FREDERICK. Map projections: theory and applications, CRC Press Inc., 1990. $372 \mathrm{p}$.

PINO, LEONARDO M.; FIRKOWSKI, HENRIQUE, Avaliação de modelos de transformação bidimensional para a compatibilização de bases cartográficas associadas a diversos sistemas geodésicos de referência, Boletim de Ciências Geodésicas, UFPR, Curitiba, v. 15, n. 2, 2009.

RAPP, H. R. Geodésica Geométrica, v. 2, Técnicas Avanzadas, Departamento de Ciencias Geodésicas, Universidad Estatal de Ohio, Columbus, 1981. 196 p.

SMITH, J. R. Introduction to geodesy: the history and concepts of modern geodesy New York: New York: J. Wiley, 1996. 224 p.

SNYDER, J. Map Projections - A Working Manual, U.S. Geological Survey Professional Paper 1395, Washington. 1987. 384 p

(Recebido em março de 2012. Aceito em outubro de 2012.) 